\title{
COMPORTAMIENTO ASINTÓTICO DE LAS SOLUCIONES DE UNA ECUACIÓN DIFERENCIAL ORDINARIA NO LINEAL DE ORDEN $n$
}

\section{Santiago César Rojas Romero ${ }^{1}$}

RESUMEN.- En este artículo demostramos que las soluciones de Ecuaciones Diferenciales Odinarias (EDO) no lineales con coeficientes continuos y términos no lineales tendiendo a cero en el infinito, tienen comportamiento asintótico similar a las soluciones de sus correpondientes ecuaciones lineales.

PALABRAS CLAVE.- Comportamiento asintótico, Desigualdad de Gronwall, E.D.O. no lineales.

\section{ASYMPTOTIC BEHAVIOR OF SOLUTIONS*OF AN n-TH ORDER NONLINEAR O.D.E.}

\begin{abstract}
In this paper, we prove that the solution of nonlinear Ordinary Differential Equations (ODE), with continuous coefficients and non-linear terms tending to zero at infinity, have an asymptotic behavior similar to the solution of there corresponding linear equations.
\end{abstract}

KEYWORDS.- Asymptotic behavior, Gronwall's inequality, Non-linear $O D E$.

\section{INTRODUCCIÓN}

Sean las ecuaciones

$$
\begin{gathered}
x^{(n)}+\sum_{i=0}^{n-1} \sum_{j=1}^{m_{i}} a_{i j}(t)\left[x^{(i)}\right]^{r_{i j}}=0, \\
x^{(n)}+b_{n-1}(t) x^{(n-1)}+\ldots+b_{1}(t) x^{\prime}+b_{0}(t) x+\sum_{i=0}^{n-1} \sum_{j=1}^{m_{i}} a_{i j}(t)\left[x^{(i)}\right]^{r_{i j}}=0 .
\end{gathered}
$$

Donde $n \geq 2$, los $m_{i}$ son arbitrarios pero finitos y todos los coeficientes $a_{i j}$ y $b_{i}$ son continuos; además cada $r_{i j}$ tiene la forma $p_{i j} / q_{i j}$ con $p_{i j}$ entero positivo y $q_{i j}$ entero positivo impar.

\footnotetext{
${ }^{1}$ Profesor de la Facultad de Ciencias Matemáticas de la Universidad Nacional Mayor de San Marcos. E-mail: santiagocesar2@hotmail.com
} 
Asumiendo que los términos no tienden a cero cuando $t$ tiende al infinito, veremos que las soluciones de (1) y (2) tienen comportamiento asintótico similar a las soluciones de las correspondientes ecuaciones lineales

$$
\begin{aligned}
x^{(n)} & =0, \\
x^{(n)}+b_{n-1}(t) x^{(n-1)}+\ldots+b_{1}(t) x^{\prime}+b_{0}(t) x & =0 .
\end{aligned}
$$

Waltman [1] prueba los resultados fundamentales usando elaboradas herramientas. Aquí agregamos algunas hipótesis y en las pruebas usaremos una versión generalizada de la desigualdad de Gronwall, la cual también incluimos en el trabajo con el ánimo de hacerlo más completo.

\section{DESIGUALDAD DE GRONWALL}

\section{Lema.}

Sean $t_{1} \geq t_{0}, f$ y $g$ continuas y no negativas para $t \geq t_{1}, t \geq t_{0}$, respectivamente. Supongamos que,

$$
f(t) \leq \delta+\int_{t_{1}}^{t} g(\tau) f^{r}(\tau) d \tau, \forall t \geq t_{1} \geq t_{0}
$$

donde $\delta>0$ y $r \geq 1$; además $\int_{t_{0}}^{\infty} g(\tau) d \tau<\infty$. Si $r>1$ y ambos $t_{0}$ y $\delta$ satisfacen

$$
\delta<\left[(r-1) \int_{t_{0}}^{\infty} g(\tau) d \tau\right]^{-1 /(r-1)}
$$

entonces $f(t)$ es acotado en $\left[t_{1}, \infty\right)$. Si $r=1$, la conclusión sigue siendo válida sin necesidad de la condición sobre to $y \delta$.

\section{Prueba.}

Pongamos $h(t)=\delta+\int_{t_{1}}^{t} g(\tau) f^{r}(\tau) d \tau$. De aquí $h^{\prime}(t)=g(t) f^{r}(t)$ y por hipótesis $f(t) \leq h(t)$. Luego, $h^{\prime}(t) \leq g(t) h^{r}(t)$ y de allí que

$$
\int_{t_{1}}^{t} h^{-r}(\tau) h^{\prime}(\tau) d \tau \leq \int_{t_{1}}^{t} g(\tau) d \tau \leq \int_{t_{0}}^{\infty} g(\tau) d \tau
$$

Si $r=1$, tenemos

$$
\ln \frac{h(t)}{h\left(t_{1}\right)} \leq \int_{t_{0}}^{\infty} g(\tau) d \tau, \quad t \geq t_{1}
$$


de donde

$$
h(t) \leq \delta \exp \left[\int_{t_{0}}^{\infty} g(\tau) d \tau\right], t \geq t_{1} .
$$

Si $r>1$, tenemos

$$
-\frac{1}{r-1}\left[h^{1-r}(t)-h^{1-r}\left(t_{1}\right)\right] \leq \int_{t_{0}}^{\infty} g(\tau) d \tau, t \geq t_{1}
$$

o lo que es lo mismo

$$
\delta^{-(r-1)}-(r-1) \int_{t_{0}}^{\infty} g(\tau) d \tau \leq h^{1-r}(t), t \geq t_{1}
$$

de donde

$$
h(t) \leq\left[\delta^{-(r-1)}-(r-1) \int_{t_{0}}^{\infty} g(\tau) d \tau\right]^{-1 /(r-1)} \quad ; t \geq t_{1} .
$$

Usando (3) tenemos que $\left[\delta^{-(r-1)}-(r-1) \int_{t_{0}}^{\infty} g(\tau) d \tau\right]>0, y$, por tanto, $h(t)$ es acotada sobre $\left[t_{1}, \infty\right)$. Finalmente, como $f(t) \leq h(t)$ sobre $\left[t_{1}, \infty\right)$, se tiene el resultado.

\section{PRINCIPALES RESULTADOS}

\section{Teorema 1.}

$$
\begin{gathered}
\int_{t_{0}}^{\infty} \sum_{i=0}^{n-1} \sum_{j=1}^{m_{i}}\left|a_{i j}(t)\right| t^{(n-1-i) r_{i j}} d t<\infty \quad y \quad r=\max \left\{r_{i j}\right\}>1 \text { verifica la desigualdad } \\
\max \left[1, \sum_{p=0}^{n-1} \sum_{k=0}^{n-1-p} \frac{\left|x^{(p+k)}\left(t_{0}\right)\right|}{k ! t_{0}^{n-1-p-k}}\right] \\
<\left[(r-1) \sum_{p=0}^{n-1} \frac{1}{p !} \int_{t_{0}}^{\infty} \sum_{i=0}^{n-1} \sum_{j=1}^{m_{i}}\left|a_{i j}(t)\right| t^{(n-1-i) r_{i j}} d t\right]^{-1 /(r-1)}
\end{gathered}
$$

para algún $t_{0}>0$. Entonces la ecuación (1) tiene soluciones $x(t)$ tales que,

$$
\lim _{t \rightarrow \infty} x^{(n-1)}(t)=\alpha>0 \quad(\alpha \text { finito }) .
$$


Si $r=\max \left\{r_{i j}\right\} \leq 1$, la conclusión sigue siendo válida sin necesidad de que la desigualdad (4) se verifique.

\section{Prueba.}

Aplicando la fórmula de Taylor con resto integral, tenemos

$$
x^{(p)}(t)=\sum_{k=0}^{n-1-p} \frac{x^{(p+k)}\left(t_{0}\right)}{k !}\left(t-t_{0}\right)^{k}+\frac{1}{(n-1-p) !} \int_{t_{0}}^{t}(t-\tau)^{n-1-p} x^{(n)}(\tau) d \tau
$$

donde, $\quad p=0,1, \ldots, n-1, t \geq t_{0}>0$

De aquí,

$$
\begin{aligned}
\frac{x^{(p)}(t)}{t^{n-1-p}} & =\sum_{k=0}^{n-1-p} \frac{x^{(p+k)}\left(t_{0}\right)}{k ! t^{n-1-p-k}}\left(\frac{t-t_{0}}{t}\right)^{k}-\frac{1}{(n-1-p) !} \\
& \cdot \int_{t_{0}}^{t}\left(\frac{t-\tau}{t}\right)^{n-1-p} \sum_{i=0}^{n-1} \sum_{j=1}^{m_{i}} a_{i j}(\tau) \tau^{(n-1-i) r_{i j}}\left[\frac{x^{(i)}(\tau)}{\tau^{n-1-i}}\right]^{r_{i j}} d \tau,
\end{aligned}
$$

de donde obtenemos la desigualdad

$$
\begin{aligned}
\frac{\left|x^{(p)}(t)\right|}{t^{n-1-p}} \leq & \sum_{k=0}^{n-1-p} \frac{\left|x^{(p+k)}\left(t_{0}\right)\right|}{k ! t_{0}^{n-1-p-k}}+\frac{1}{(n-1-p) !} \\
& \cdot \int_{t_{0}}^{t} \sum_{i=0}^{n-1} \sum_{j=1}^{m_{i}}\left|a_{i j}(\tau)\right| \tau^{(n-1-i) r_{i j}}\left\{\max \left[1, \sum_{k=0}^{n-1} \frac{\left|x^{(k)}(\tau)\right|}{\tau^{n-1-k}}\right]\right\}^{r} d \tau .
\end{aligned}
$$

Sumando sobre $p$ tenemos

$$
\begin{aligned}
\max [1, & \left.\sum_{p=0}^{n-1} \frac{\left|x^{(p)}(t)\right|}{t^{n-1-p}}\right] \leq \max \left[1, \sum_{p=0}^{n-1} \sum_{k=0}^{n-1-p} \frac{\left|x^{(p+k)}\left(t_{0}\right)\right|}{k ! t_{0}^{n-1-p-k}}\right] \\
& +\sum_{p=0}^{n-1} \frac{1}{p !} \int_{t_{0}}^{t} \sum_{i=0}^{n-1} \sum_{j=1}^{m_{i}}\left|a_{i j}(\tau)\right| \tau^{(n-1-i) r_{i j}}\left\{\max \left[1, \sum_{k=0}^{n-1} \frac{\left|x^{(k)}(\tau)\right|}{\tau^{n-1-k}}\right]\right\}^{r} d \tau
\end{aligned}
$$

Del lema previo, con

$$
f(t)=\max \left[1, \sum_{p=0}^{n-1} \frac{\left|x^{(p)}(t)\right|}{t^{n-1-p}}\right] y \quad g(t)=\sum_{p=0}^{n-1} \frac{1}{p !} \sum_{i=0}^{n-1} \sum_{j=1}^{m_{i}}\left|a_{i j}(t)\right| t^{(n-1-i) r_{i j}}
$$


se sigue que $\max \left[1, \sum_{p=0}^{n-1} \frac{\left|x^{(p)}(t)\right|}{t^{n-1-p}}\right]$ es acotado sobre $\left[t_{0}, \infty\right)$.

Ahora, como

$$
x^{(n-1)}(t)=x^{(n-1)}\left(t_{0}\right)-\int_{t_{0}}^{t} \sum_{i=0}^{n-1} \sum_{j=1}^{m_{i}} a_{i j}(\tau) \tau^{(n-1-i)} r_{i j}\left[\frac{\left|x^{(i)}(\tau)\right|}{\tau^{n-1-i}}\right]^{r_{i j}} d \tau,
$$

se tiene que $\lim _{t \rightarrow \infty} x^{(n-1)}(t)$ existe. Queda por demostrar que siempre existen soluciones $x(t)$ tales que este límite es mayor que cero.

Escogemos $t_{0}>0, \delta \geq 1$. Si $r>1$ requerimos adicionalmente que $t_{0}$ y $\delta$ verifiquen

$$
\delta<\left[(r-1) \sum_{p=0}^{n-1} \frac{1}{p !} \int_{t_{0}}^{\infty} \sum_{i=0}^{n-1} \sum_{j=1}^{m_{i}}\left|a_{i j}(t)\right| t^{(n-1-i) r_{i j}} d t\right]^{-1 /(r-1)} .
$$

Por el lema, existe una cota $M$ tal que, si $y(t)$ es cualquier función continua no negativa verificando

$$
y(t) \leq \delta+\sum_{p=0}^{n-1} \frac{1}{p !} \int_{t_{1}}^{t} \sum_{i=0}^{n-1} \sum_{j=1}^{m_{i}}\left|a_{i j}(\tau)\right| \tau^{(n-1-i) r_{i j}} y^{r}(\tau) d \tau
$$

para todo $t \geq t_{1} \geq t_{0}$, entonces $y(t) \leq M$ sobre $\left[t_{1}, \infty\right)$.

Escogemos $t_{1}$ suficientemente grande tal que,

$$
\int_{t_{1}}^{\infty} \sum_{i=0}^{n-1} \sum_{j=1}^{m_{i}}\left|a_{i j}(t)\right| t^{(n-1-i) r_{i j}} d t<\frac{\delta}{M^{r} \sum_{p=0}^{n-1} \frac{1}{p !}}
$$

Sea $x(t)$ la solución de (1) satisfaciendo las condiciones iniciales $x\left(t_{1}\right)=x^{\prime}\left(t_{1}\right)=\ldots=x^{(n-2)}\left(t_{1}\right)=0, x^{(n-1)}\left(t_{1}\right)=\delta / \sum_{p=0}^{n-1} \frac{1}{p !}$. Procediendo como antes obtenemos la desigualdad (5) con $t_{1}$ en lugar de $t_{0}$. Puesto que

$$
\max \left[1, \sum_{p=0}^{n-1} \sum_{k=0}^{n-1-p} \frac{\left|x^{(p+k)}\left(t_{1}\right)\right|}{k ! t^{n-1-p-k}}\right]=\delta
$$




\section{COMPORTAMIENTO ASINTÓTICO DE LAS ...}

sigue del lema que $\max \left[1, \sum_{p=0}^{n-1} \frac{\left|x^{(p)}(t)\right|}{t^{n-1-p}}\right]$ es acotado por $M$ en $\left[t_{1}, \infty\right)$.

De aquí

$$
\begin{aligned}
& \lim _{t \rightarrow \infty} x^{(n-1)}(t)=x^{(n-1)}\left(t_{1}\right)-\int_{t_{1}}^{\infty} \sum_{i=0}^{n-1} \sum_{j=1}^{m_{i}} a_{i j}(\tau) \tau^{(n-1-i)} r_{i j}\left[\frac{x^{(i)}(\tau)}{\tau^{n-1-i}}\right]^{r_{i j}} d \tau \\
& \geq \frac{\delta}{\sum_{p=0}^{n-1} \frac{1}{p !}}-M^{r} \int_{t_{1}}^{\infty} \sum_{i=0}^{n-1} \sum_{j=1}^{m_{i}}\left|a_{i j}(t)\right| t^{(n-1-i) r_{i j}} d t>0 .
\end{aligned}
$$

Esto completa la prueba. (2).

Ahora centramos nuestra atención en la ecuación más general dada en

\section{Teorema 2.}

Consideremos la ecuación (2). Sean $x_{1}(t), x_{2}(t), \ldots, x_{n}(t)$ soluciones linealmente independientes de la ecuación lineal asociada $\left(2^{*}\right)$ y $W(t)$ su Wronskiano. Sea

$$
\begin{gathered}
X_{i}(t)=\max _{1 \leq k \leq n}\left|x_{k}^{(t)}\right|, i=0,1, \ldots, n-1, y \text { sean } \\
W_{k}(t)=\text { Wronskiano }\left[x_{1}(t), \ldots, x_{k-1}(t), x_{k+1}(t), \ldots, x_{n}(t)\right], k=1,2, \ldots, n \quad \mathrm{y} \\
Z(t)=\max _{1 \leq k \leq n}\left|W_{k}(t)\right| .
\end{gathered}
$$

Si

$$
\int_{t_{0}}^{\infty} \frac{Z(t)}{|W(t)|} \sum_{i=0}^{n-1} \sum_{j=1}^{m_{i}}\left|a_{i j}(t)\right| X_{i}^{r_{i j}} d t<\infty
$$

Entonces, la ecuación (2) tiene soluciones $x(t)$ (no triviales), tales que

$$
x^{(p)}(t)=\sum_{k=1}^{n} A_{k}(t) x_{k}^{(p)}(t), \quad p=0,1, \ldots, n-1
$$

Donde las funciones $A_{k}(t)$ son tales que

$$
\lim _{t \rightarrow \infty} A_{k}(t)=a_{k} \text { (finito) }, k=1,2, \ldots, n \text {. }
$$


Si $r=\max \left\{r_{i j}\right\}>1$, adicionalmente necesitamos que se verifique la desigualdad

$$
\begin{aligned}
\max \left[1, \sum_{p=1}^{n}\left|A_{p}\left(t_{0}\right)\right|\right] & \\
< & {\left[n(r-1) \int_{t_{0}}^{\infty} \frac{Z(t)}{|W(t)|} \sum_{i=0}^{n-1} \sum_{j=1}^{m_{i}}\left|a_{i j}(t)\right| X_{i}^{r_{i j}}(t) d t\right]^{-1 /(r-1)} }
\end{aligned}
$$

para algún $t_{0}$.

\section{Prueba.}

Sea $x(t)$ una solución de (2). Sean $A_{k}(t)$ las funciones definidas (únicamente) en términos de $x(t), x^{\prime}(t), \ldots, x^{(n-1)}(t)$ por las ecuaciones (7).

De las ecuaciones (7) y el hecho que $x(t)$ es una solución de (2) obtenemos las ecuaciones

$$
\begin{aligned}
& \sum_{k=1}^{n} A_{k}^{\prime}(t) x_{k}^{(p)}(t)=0, p=0,1, \ldots, n-2 \\
& \sum_{k=1}^{n} A_{k}^{\prime}(t) x_{k}^{(n-1)}(t)=-\sum_{i=0}^{n-1} \sum_{j=1}^{m_{i}} a_{i j}(t)\left[\sum_{k=1}^{n} A_{k}(t) x_{k}^{(i)}(t)\right]^{r_{i j}} \cdot
\end{aligned}
$$

Resolviendo este sistema para $A_{1}^{\prime}(t), A_{2}(t), \ldots, A_{n}^{\prime}(t)$ e integrando de $t_{0}$ a $t$, tenemos

$$
\begin{aligned}
A_{p}(t)=A_{p}\left(t_{0}\right)+ & (-1)^{n+p+1} \int_{t_{0}}^{t} \frac{W_{p}(\tau)}{W(\tau)} \sum_{i=0}^{n-1} \sum_{j=1}^{m_{i}} a_{i j}(\tau) \\
& \cdot\left[\sum_{k=1}^{n} A_{k}(\tau) x_{k}^{(i)}(\tau)\right]^{r_{i j}} d \tau, p=1,2, \ldots, n
\end{aligned}
$$

de donde obtenemos la desigualdad

$$
\begin{aligned}
\max \left[1, \sum_{p=1}^{n}\left|A_{p}(t)\right|\right] & \leq \max \left[1, \sum_{p=1}^{n}\left|A_{p}\left(t_{0}\right)\right|\right] \\
& +n \int_{t_{0}}^{t} \frac{Z(\tau)}{|W(\tau)|} \sum_{i=0}^{n-1} \sum_{j=1}^{m_{i}}\left|a_{i j}(\tau)\right| X_{i}^{r_{i j}}(\tau) \\
& \cdot\left\{\max \left[1, \sum_{k=1}^{n}\left|A_{k}(\tau)\right|\right]\right\}^{-r} d \tau
\end{aligned}
$$




\section{COMPORTAMIENTO ASINTÓTICO DE LAS ...}

Por el lema tenemos que max $\left[1, \sum_{p=1}^{n}\left|A_{p}(t)\right|\right]$ es acotado sobre $\left[t_{0}, \infty\right)$. De ahí que las integrales en las ecuaciones (10) son absolutamente convergentes cuando $t \rightarrow \infty$, de este modo concluimos que $\lim _{t \rightarrow \infty} A_{p}(t)$ existe (finito), $p=1,2, \ldots, n$.

\section{Teorema 3.}

Con las mismas hipótesis del teorema 2, y adicionalmente asumiendo que:

i) Si $x_{k}^{(p)}(t)$ no es acotado cuando $t \rightarrow \infty$, entonces existe una función (positiva) continua y no decreciente $x_{k p}^{*}(t)$ tal que $\left|x_{k}^{(p)}(t)\right| \leq x_{k p}^{*}(t)$ para $t$ sufientemente grande.

ii)

$$
\int_{t_{0}}^{\infty} \frac{x_{k p}^{*}(t)\left|W_{k}(t)\right|}{|W(t)|} \sum_{i=0}^{n-1} \sum_{j=1}^{m_{i}}\left|a_{i j}(t)\right| X_{i}^{r_{i j}}(t) d t<\infty
$$

Entonces, la ecuación (2) tiene soluciones (no triviales) $x(t)$ tales que,

$$
x^{(p)}(t)=\sum_{k=1}^{n} a_{k} x_{k}^{(p)}(t)+\varepsilon^{(p)}(t), p=0,1, \ldots, n-1
$$

donde los $a_{k}$ son constantes $y \lim _{t \rightarrow \infty} \varepsilon^{(p)}(t)=0$.

\section{Prueba.}

Usamos las notaciones y resultados del Teorema 3. En primer lugar, establecemos que

$$
\lim _{t \rightarrow \infty}\left|A_{k}(t)-a_{k}\right|\left|x_{k}^{(p)}(t)\right|=0
$$

Esto se da obviamente cuando $x_{k}^{(p)}(t)$ es acotado, así que asumimos que $x_{k}^{p}$ no es acotado. Resolviendo para $A_{k}^{\prime}(t)$ en (9) y luego integrando de $t$ a $\infty$ tenemos

$$
a_{k}=A_{k}(t)+(-1)^{n+k+1} \int_{t}^{\infty} \frac{W_{k}(\tau)}{W(\tau)} \sum_{i=0}^{n-1} \sum_{j=1}^{m_{i}} a_{i j}(\tau)\left[\sum_{k=0}^{n} A_{k}(\tau) x_{k}^{(i)}(\tau)\right]^{r_{i j}} d \tau
$$

De aqui se tiene que para $t$ suficientemente grande, 


$$
\begin{aligned}
\left|A_{k}(t)-a_{k}\right|\left|x_{k}^{(p)}(t)\right| \leq & \int_{t}^{\infty} \frac{x_{k p}^{*}(\tau)\left|W_{k}(\tau)\right|}{|W(\tau)|} \sum_{i=0}^{n-1} \sum_{j=1}^{m_{i}}\left|a_{i j}(\tau)\right| X_{i}^{r_{i j}}(\tau) \\
& \cdot\left\{\max \left[1, \sum_{k=1}^{n}\left|A_{k}(\tau)\right|\right]\right\}^{r} d \tau .
\end{aligned}
$$

De lo anterior se tiene que

$$
\lim _{t \rightarrow \infty}\left|A_{k}(t)-a_{k}\right|\left|x_{k}^{(p)}(t)\right|=0
$$

donde

$$
x^{(p)}(t)=\sum_{k=1}^{n} A_{k}(t) x_{k}^{(p)}(t)=\sum_{k=1}^{n} a_{k} x_{k}^{(p)}(t)+\varepsilon_{p}(t) .
$$

Donde $\lim _{t \rightarrow \infty} \varepsilon_{p}(t)=0$. Poniendo $\varepsilon_{0}(t)=\varepsilon(t)$, tenemos $\varepsilon_{p}(t)=\varepsilon^{(p)}(t)$, con lo cual el teorema queda probado.

Aplicando el Teorema 3 a la ecuación (1) tenemos el siguiente resultado.

\section{Teorema 4.}

Si

$$
\int_{t_{0}}^{\infty} t^{n-1} \sum_{i=0}^{n-1} \sum_{j=1}^{m_{i}}\left|a_{i j}(t)\right| t^{(n-1-i) r_{i j}} d t<\infty
$$

entonces (1) tiene soluciones (no triviales) $x(t)$ tales que,

$$
x(t)=\sum_{k=1}^{n} a_{k} t^{k-1}+\varepsilon(t),
$$

donde

$$
\lim _{t \rightarrow \infty} \varepsilon^{(p)}(t)=0, p=0,1, \ldots, n-1 .
$$

Prueba.

Utilizando la notación de los Teoremas 2 y 3 tenemos $x_{k}(t)=t^{k-1}, k=1,2, \ldots, n$ y $W(t)=$ constante. Podemos tomar $X_{i}(t)=(n-1) ! t^{n-1-i}$ para $t \geq 1$ $i=0,1, \ldots, n-1 . W_{k}(t)$ es la forma $c_{k} t^{n-k}$ y $Z(t)=c t^{n-1}$ para $n \geq 1$. Facilmente se observa que, todas las hipótesis del Teorema 3 se verifican, por lo tanto, el teorema esta probado. 


\section{REFERENCIAS BIBLIOGRÁFICAS}

[1] Waltman Paul. On the asymptotic behavior of solutions of a nonlinear equation. Proc. Amer. Math. Soc. 15, pag. 918 - 923 (1964).

[2] Locke Phill. On the asymptotic behavior of solutions of an nth-order nonlinear equation Proc. Amer. Math. Soc. 18, pag. 383 - 390 (1967). 